Supporting Information

\title{
Superhydrophobic and Superoleophilic Micro-Wrinkled Reduced Graphene Oxide as a Highly Portable and Recyclable Oil Sorbent
}

Chunfang Feng, ${ }^{\text {a } Z h i f e n g ~ Y i, ~}{ }^{a}$ Fenghua She, ${ }^{a}$ Weimin Gao, ${ }^{a}$ Zheng Peng, ${ }^{\text {a,b }}$ Christopher J. Garvey, Ludovic F. Dumée ${ }^{\mathrm{a}}$ and Lingxue Kong ${ }^{*}$,a

${ }^{\mathrm{a}}$ Deakin University, Geelong, Victoria 3216, Australia - Institute for Frontier Materials

${ }^{\mathrm{b}}$ Agricultural Product Processing Research Institute (APPRI), Chinese Academy of Tropical Agricultural Sciences (CATAS), Zhanjiang 524001, China

${ }^{\mathrm{c}}$ Australian Nuclear Science and Technology Organisation (ANSTO), Lucas Heights, New South Wales 2234, Australia

*Corresponding author: Lingxue Kong (Tel: +61 352272087 and E-mail:

lingxue.kong@deakin.edu.au). 
Table S1 Physical properties of the oils tested in this work

\begin{tabular}{lll}
\hline Oil Types & Density $(\mathrm{g} / \mathrm{mL})$ & Viscosity $\left(\mathrm{cSt}, @ 20{ }^{\circ} \mathrm{C}\right)$ \\
\hline Pump Oil & 0.87 & 55 \\
Canola Oil & 0.91 & 70 \\
Engine Oil & 0.88 & 550 \\
\hline
\end{tabular}


Table S2 Water sorption of MWrGO samples with different GO thicknesses

\begin{tabular}{ll}
\hline Samples & Water Sorption $(\mathrm{g} / \mathrm{g})$ \\
\hline MWrGO-1 & $0.51 \pm 0.11$ \\
MWrGO-2 & $0.49 \pm 0.13$ \\
MWrGO-3 & $0.52 \pm 0.10$ \\
\hline
\end{tabular}



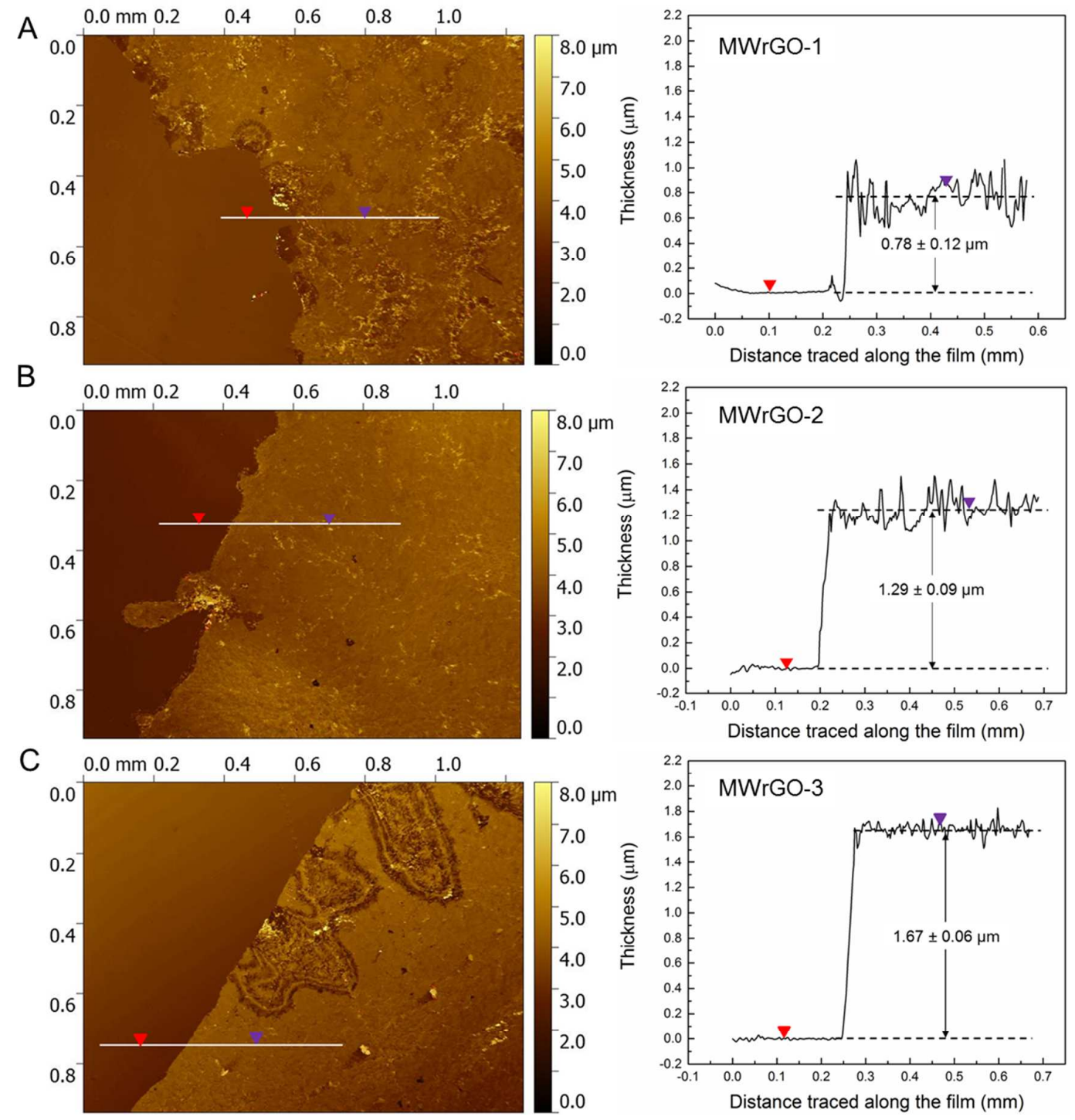

Figure S1 2D graphs of GO film with different thickness on the shrinking film (left) and the corresponding typical film thickness profiles recorded by profilometer during single trace along $\mathrm{X}$ axis (right). (A) MWrGO-1, (B) MWrGO-2 and (C) MWrGO-3. 

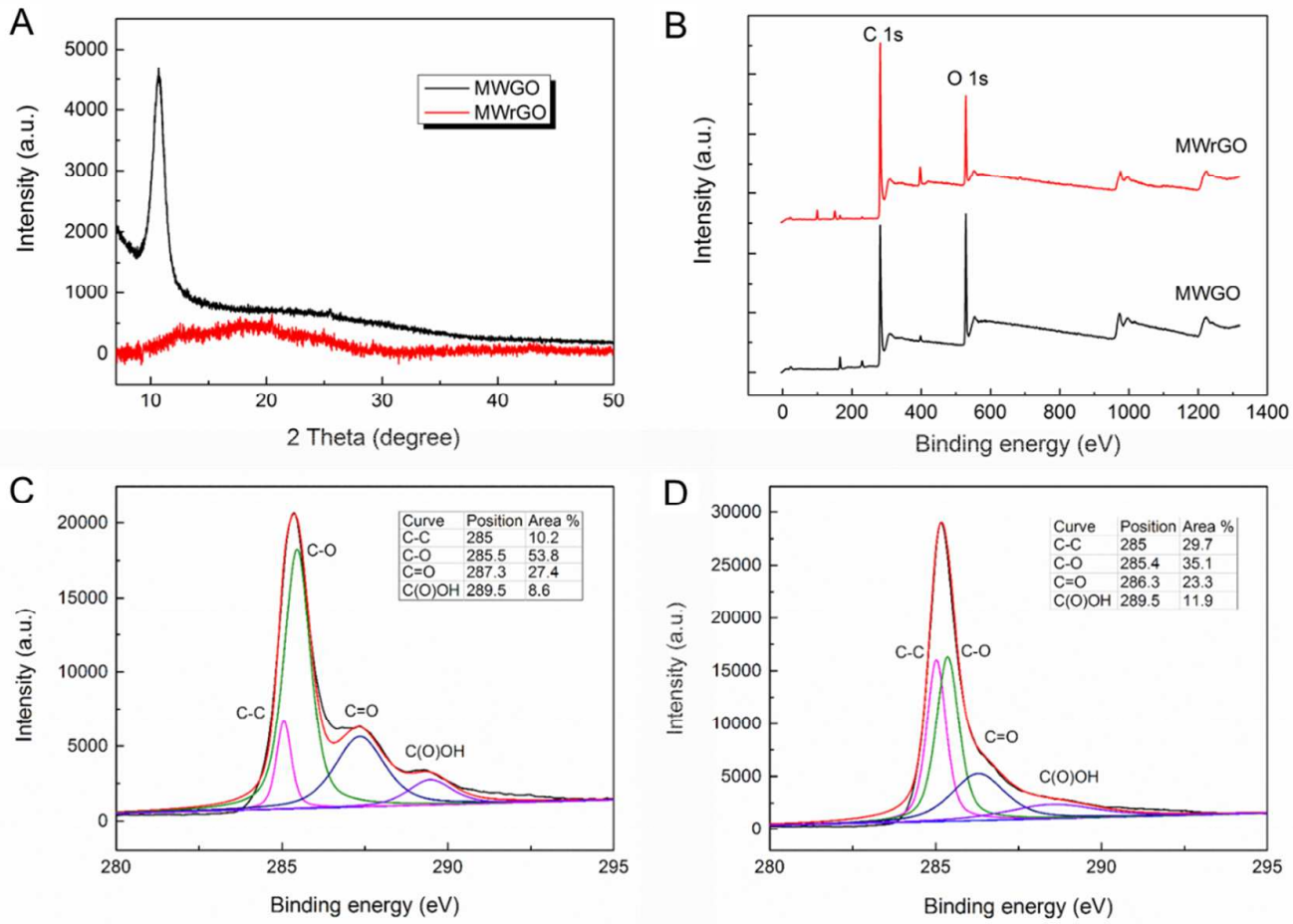

Figure S2 (A) XRD spectrum: the characteristic peak at $2 \vartheta$ of $11^{\circ}$ is the representative scattering from rGO sheets. (B) XPS spectrum of low resolution scan. (C) and (D) XPS spectra of high resolution scan for MWGO and MWrGO respectively. The obvious decease in oxygen content can be seen after chemical reduction. 

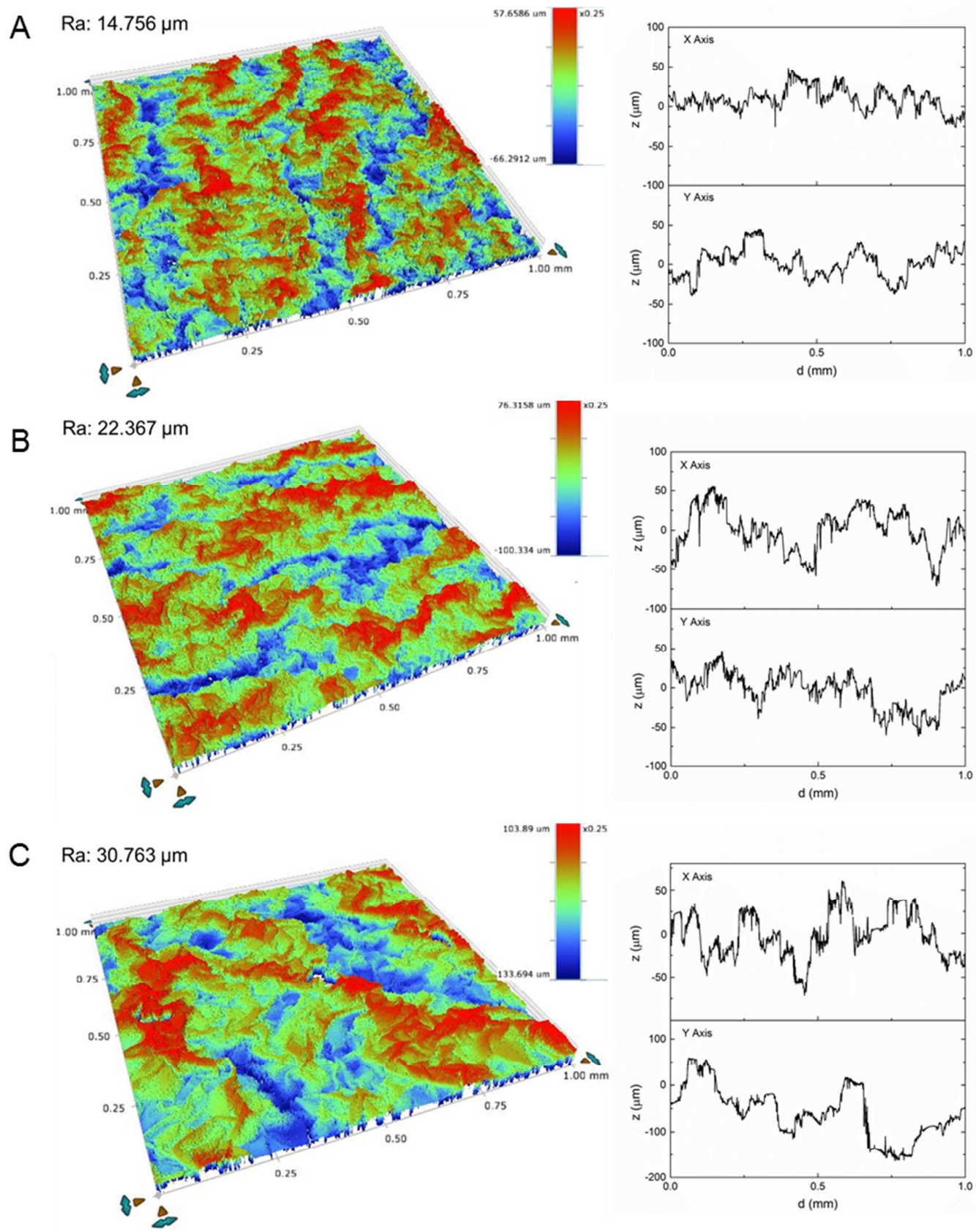

Figure S3 Representative roughness profiles of MWrGO-1 (A), MWrGO-2 (B) and MWrGO-3 (C). $R_{a}$ is the calculated average roughness. 

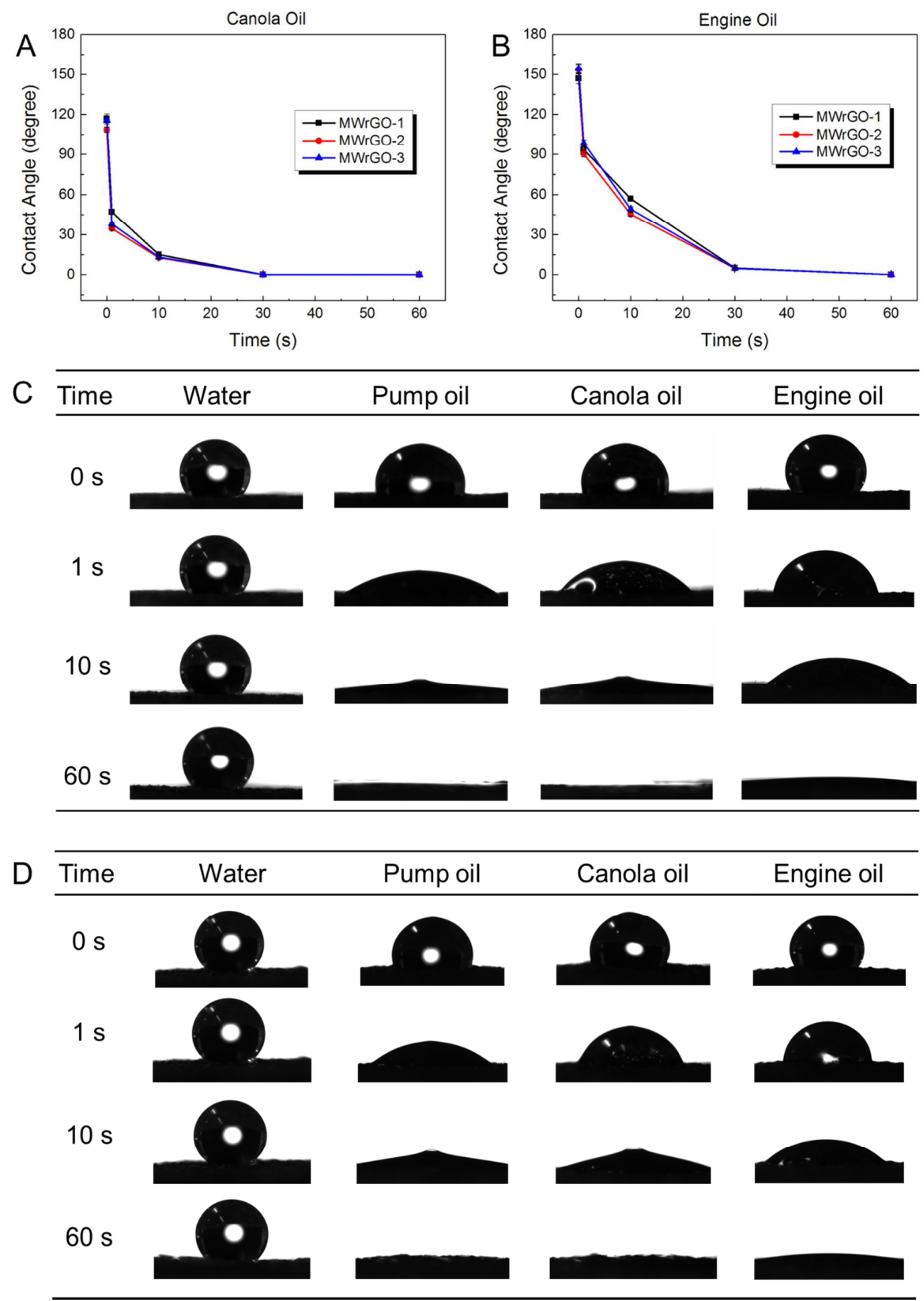

Figure S4 Changes of contact angles against time on MWrGO-1, MWrGO-2 and MWrGO-3 for canola oil (A) and engine oil (B). Photographs of water and oil droplets on the surface of MWrGO. (C) MWrGO-2 and (D) MWrGO-3. 

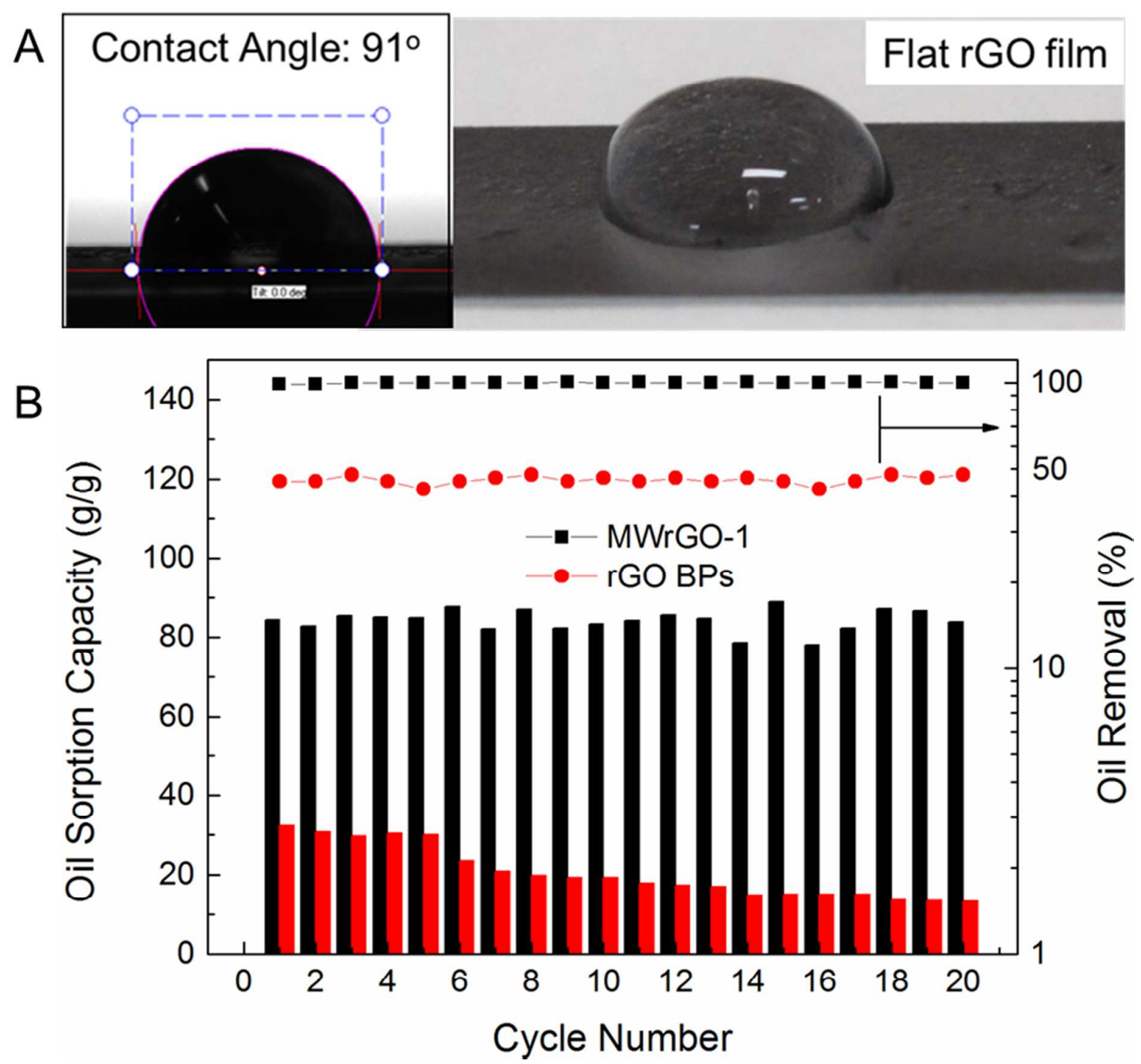

Figure S5 (A) Water contact angle of flat rGO film (left) and the photo of water droplet on the surface of flat rGO film (right). (B) Comparison of oil sorption capacity and oil removal efficiency of engine oil between rGO BPs and MWrGO-1 sample. 